\title{
Análisis poscosecha de frutos de pitahaya amarilla (Cereus triangularis Haw.), a distintos niveles de madurez y temperatura
}

Post-harvest analysis of yellow pitahaya fruits (Cereus triangularis Haw.) At different maturity and temperature levels

\author{
Jiménez-Esparza Luis Oswaldo ${ }^{*}$, González-Parra Marilú Manuela², Cruz-Tobar Saúl Eduardo², \\ Santana-Mayorga Rita ${ }^{2}$, Villacís Aldaz Luis Alfredo ${ }^{2}$
}

\begin{tabular}{|c|c|}
\hline Datos del Artículo & Resumen \\
\hline 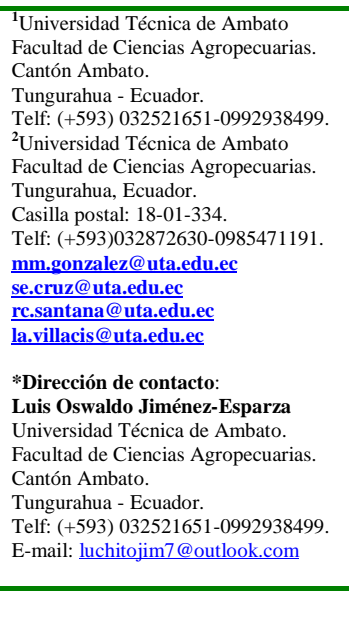 & $\begin{array}{l}\text { Las frutas de pitahaya amarilla (Cereus triangularis Haw.), tienden a sufrir un rápido deterioro una vez cosechadas. Con } \\
\text { el objetivo de estudiar las condiciones óptimas de almacenamiento para preservar su vida útil, se evaluó la calidad posco- } \\
\text { secha de la pitahaya amarilla en almacenamiento, combinando la temperatura (factor A) con dos niveles: A1(ambiente) } \\
16 \pm 4{ }^{\circ} \mathrm{C} \text { y HR de } 79 \% \text {, A2 (frío) } 6 \pm 2{ }^{\circ} \mathrm{C} \text { y HR de } 72-92 \% \text {, y el estado fisiológico (factor B), con tres niveles: B1 fruto } \\
\text { tierno ( } 15-25 \% \text { color amarillo), B2 fruto pintón ( } 50 \% \text { color amarillo), y B3 fruto maduro ( } 75-90 \% \text { color amarillo). Se } \\
\text { estudiaron las características físico-químicas con un tiempo máximo de } 26 \text { días de duración del ensayo y las determina- } \\
\text { ciones se hicieron dos veces por semana: pérdida de peso (PP), sólidos solubles totales (SST), potencial de hidrógeno } \\
\text { (pH), acidez titulable (AT) e índice de madurez (IM). Las variables fueron comparadas mediante una prueba de media } \\
\text { según Tukey (p 0.05). No se observaron diferencias significativas en cuanto a PP (13.5\% al ambiente y } 9.3 \% \text { en frio en } \\
\text { promedio) y SST entre los tratamientos ( } 18.8 \text { promedio al ambiente y } 18.6 \text { promedio en frio). Las frutas de pitahaya } \\
\text { almacenadas al frío y con un estado de madurez pintona (A2B1) tuvieron un menor pH (4.77) existiendo diferencia entre } \\
\text { los tratamientos al ambiente (factor A) y en frio (factor B), una mayor AT tuvo (A2B1) con 3.02 con diferencia significa- } \\
\text { tiva entre el factor A y B, el menor IM en el día } 0 \text { tuvo el tratamiento A1B1 con 8.93, llegando a los } 26 \text { días a 20.20, } \\
\text { existiendo diferencia significativa entre los tratamientos del factor A y el B. }\end{array}$ \\
\hline \multirow[t]{2}{*}{$\begin{array}{l}\text { Palabras clave: } \\
\text { Calidad, } \\
\text { almacenamiento, } \\
\text { acidez titulable, } \\
\text { sólidos solubles, } \\
\text { índice de madurez. }\end{array}$} & (C) 2017. Journal of the Selva Andina Biosph. Bolivia. Todos los derechos reservados. \\
\hline & Abstract \\
\hline $\begin{array}{l}\text { J Selva Andina Biosph. } \\
\text { 2017; 5(2):107-115. }\end{array}$ & $\begin{array}{l}\text { The fruits of yellow pitahaya (Cereus triangularis Haw.), Tend to suffer rapid deterioration once harvested. In order to } \\
\text { study the optimum storage conditions to preserve its useful life, the postharvest quality of the yellow pitahaya in storage }\end{array}$ \\
\hline $\begin{array}{l}\text { Historial del artículo. } \\
\text { Recibido mayo, } 2017 . \\
\text { Devuelto agosto } 2017 \\
\text { Aceptado septiembre, } 2017 . \\
\text { Disponible en línea, noviembre } 2017 . \\
\end{array}$ & $\begin{array}{l}\text { was evaluated, combining the temperature (factor A) with two levels: A1 (ambient) } 16 \pm 4{ }^{\circ} \mathrm{C} \text { and } \mathrm{HR} \text { of } 79 \% \text {, A2 (cold) } \\
6 \pm 2{ }^{\circ} \mathrm{C} \text { and } \mathrm{HR} \text { of } 72-92 \% \text {, and the physiological state (factor B), with three levels: B1 tender fruit (15-25\% yellow } \\
\text { color), B2 pinton fruit }(50 \% \text { yellow), and B3 ripe fruit ( } 75-90 \% \text { yellow). The physico-chemical characteristics were } \\
\text { studied with a maximum time of } 26 \text { days of the test and the determinations were made twice a week: weight loss (PP), } \\
\text { total soluble solids (SST), hydrogen potential }(\mathrm{pH}) \text {, acidity titulable (AT) and maturity index (IM). The variables were }\end{array}$ \\
\hline $\begin{array}{l}\text { Editado por: } \\
\text { Selva Andina } \\
\text { Research Society }\end{array}$ & $\begin{array}{l}\text { compared by means of an average test according to Tukey ( } \mathrm{p} \text { 0.05). No significant differences were observed in PP } \\
\text { (13.5\% to the environment and } 9.3 \% \text { in cold on average) and SST among the treatments ( } 18.8 \text { average to the environ- } \\
\text { ment and } 18.6 \text { average in cold). The fruits of pitahaya stored in the cold and with a state of mature pintona (A2B1) had a } \\
\text { lower } \mathrm{pH}(4.77) \text {, there being a difference between the treatments to the environment (factor A) and cold (factor B), a }\end{array}$ \\
\hline $\begin{array}{l}\text { Quality, } \\
\text { storage, } \\
\text { titratable acidity, }\end{array}$ & $\begin{array}{l}\text { higher AT had ( A2B1) with } 3.02 \text { with a significant difference between factor A and B, the lowest MI on day } 0 \text { had the } \\
\text { A1B1 treatment with } 8.93 \text {, reaching } 26 \text { days at } 20.20 \text {, there being a significant difference between the treatments of } \\
\text { factor A and B. }\end{array}$ \\
\hline
\end{tabular}

(C) 2017. Journal of the Selva Andina Biosph. Bolivia. All rights reserved. 


\section{Introducción}

En Ecuador se identifican 2 tipos de pitahaya. En este caso, la pitahaya amarilla se cultiva en el callejón interandino y la misma variedad proveniente de la Amazonía, mientras que la roja se cultiva en la costa ecuatoriana, sin embargo se conoce la presencia de la pitahaya amarilla (Cereus sp.) con una variante que presenta las mismas características conocida también como pitahaya amarilla (Hylocereus sp.) en provincias como: Pichincha, Morona Santiago y Loja. (Pro Ecuador 2016).

La pitahaya amarilla cultivado en Ecuador es visto como una nueva alternativa de producción agrícola, elevando el nivel de vida de los agricultores (Aguilar 2015). Con el fin de desplegar una mayor diversificación en el mercado internacional, entidades públicas como PRO ECUADOR y el Ministerio de Comercio Exterior han llevado a cabo alternativas como ferias y boletines en Países de Asia y la Unión Europea, para dar a conocer la pitahaya como una fruta con alto potencial de comercialización e industrialización (Huachi et al. 2014).

Las investigaciones relacionadas con el almacenamiento postcosecha son escasas. Según (Corrales 2002), se sabe poco acerca de las posibles variaciones en el comportamiento postcosecha, así como de los parámetros de calidad de las diferentes especies cultivadas.

Los parámetros utilizados en investigaciones para determinar la calidad de la fruta, según (Balderas et al. 2016) son: peso total del fruto, peso de la cáscara (pericarpio), peso de la pulpa y semilla, sólidos solubles totales (SST) en el jugo, $\mathrm{pH}$ y acidez titulable (relación ${ }^{\circ}$ Brix/Acidez), porcentaje de azúcares totales, Vitamina $\mathrm{C}$, contenido de fenoles totales, capacidad antioxidante (DPPH) y color.
Entre los factores del manejo tras la cosecha, los más importantes son grado de madurez al momento de la cosecha, temperatura, humedad relativa, composición de la atmósfera, entre otros, en estudios realizados por (Magaña-Benítez et al. 2013) evaluaron las variaciones bioquímica- -fisiológicas y de atributos de calidad de las frutas de pitahaya, cosecharon y almacenaron a temperatura ambiente hasta 6 días, haciéndose las evaluaciones el día 0,3 y 6 días, y los resultados es que hubo pérdida de peso que está asociado a la pérdida de agua, los sólidos solubles totales (SST) no registraron variación significativa. (Osuna-Enciso et al. 2011) evaluaron la calidad postcosecha de frutos de pitahaya roja, e indicaron que los frutos cosechados en madurez media y completa mantuvieron mayores sólidos solubles totales que los frutos cosechados en madurez inicial, pero la rápida disminución de la acidez afectó su calidad. (Dueñas et al. 2009) evaluaron la calidad de frutos almacenados a temperatura ambiente y observaron que se deterioraron marcadamente tras dos semanas de almacenamiento. (Balois et al. 2013), realizaron estudios de almacenamiento de frutos de pitahaya a tres temperaturas post cosecha e informaron de que los cambios que dependieron de la temperatura y el tiempo de almacenamiento no siguieron tendencias identificables. (Rodríguez-Rodríguez et al. 2005), aconsejaron cosechar la fruta de pitahaya amarilla en estado avanzado de madurez y almacenarla a temperatura entre 8 y 19 ${ }^{\circ} \mathrm{C}$.

En base a los estudios anteriores y teniendo en cuenta que existen pocos reportes hasta la fecha de este tipo de estudio en el Ecuador, el objetivo de la investigación fue evaluar el comportamiento postcosecha de la pitahaya amarilla en diferentes grados de madurez y condiciones de almacenamiento, para 
mantener la calidad de la fruta y lograr mayores éxitos en la comercialización. (RodríguezRodríguez et al. 2005) evaluaron la vida útil de la fruta, siendo la mayor, a temperatura de $8{ }^{\circ} \mathrm{C}$. El estado de madurez de la fruta fue el factor que determinó el comportamiento poscosecha de algunos indicadores como: $\mathrm{pH}$, sólidos solubles totales ( ${ }^{\circ}$ Brix), la acidez titulable, el índice de madurez.

\section{Materiales y métodos}

Figura 1 La pitahaya amarilla "tierna", presenta una coloración Munsell 2.5GY 6/10 en el 15-25 \% de su superficie. A la derecha, malla semitransparente empleada para la determinación de color
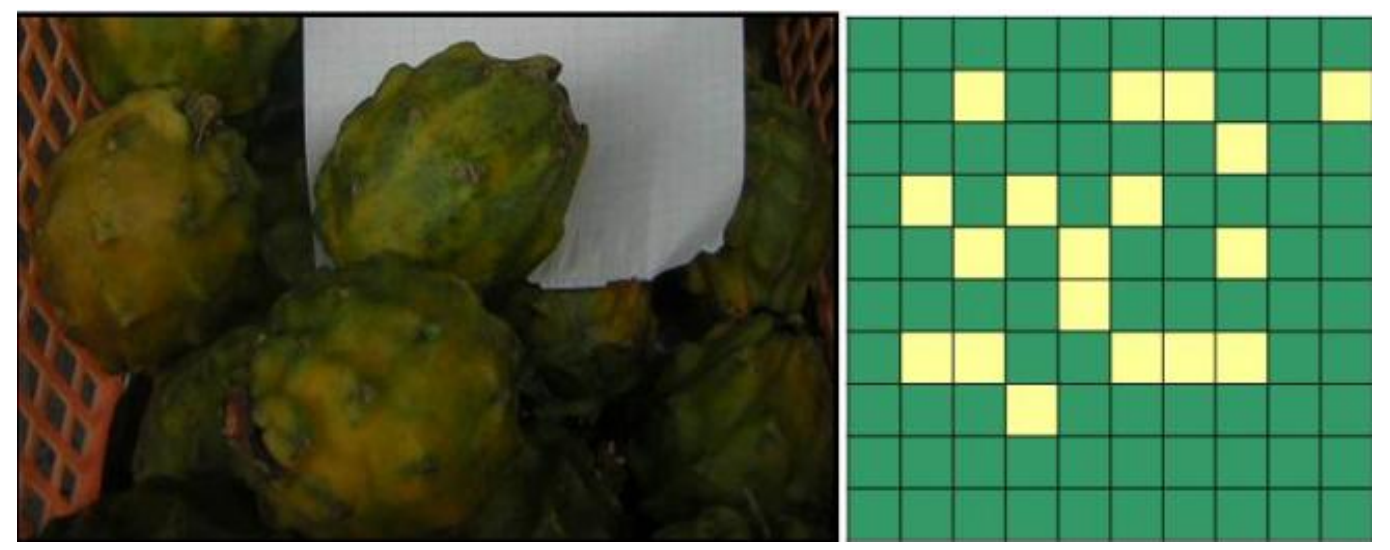

Figura 2 La pitahaya amarilla "pintona" presenta una coloración Munsell 5Y 7/10 en el $50 \%$ de su superficie. A la derecha, malla semitransparente empleada para la determinación de color

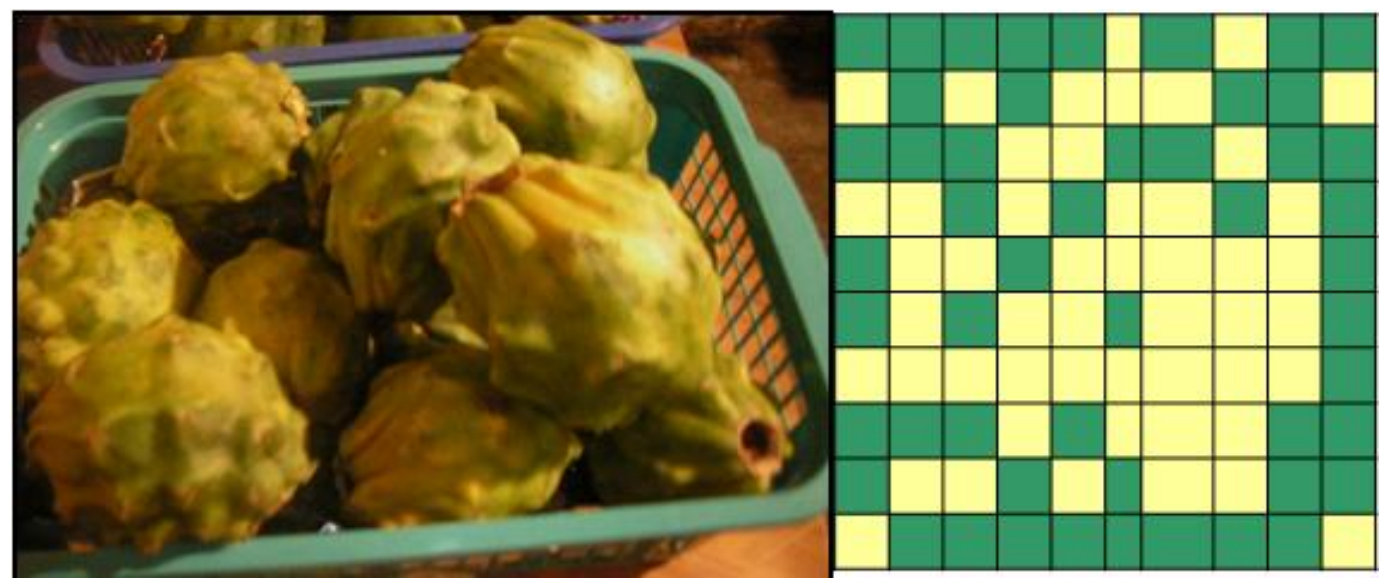


Figura 3 La pitahaya amarilla "madura" presenta una coloración Munsell 5Y 8/10 en el 75-90 \% de su superficie. A la derecha, malla semitransparente empleada para la determinación de color
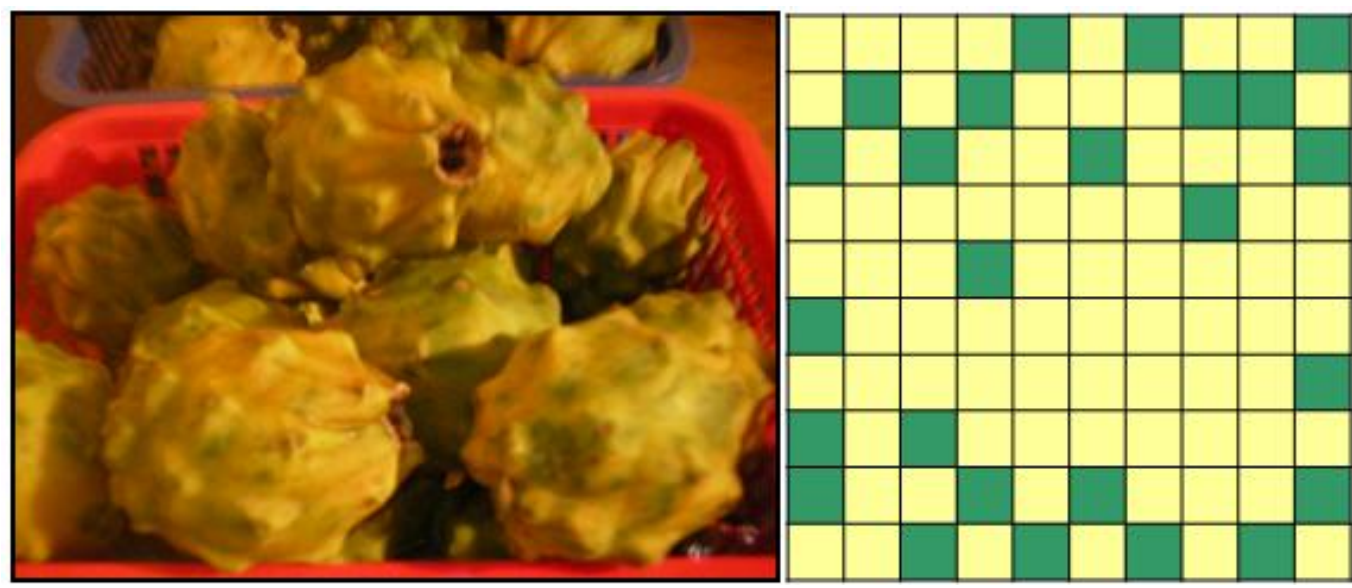

La cosecha de los frutos $(22.74 \mathrm{~kg})$ se realizó en el mes de Marzo, que es el período de producción mas alto en la Amazonía. Estos fueron cosechados manualmente y se transportaron inmediatamente en cajas de cartón al laboratorio de alimentos de la Univversidad Técnica de Ambato, para realizar los diferentes análisis.

Se aplicó un diseño de bloques completos al azar en arreglo factorial $2 \times 3$, el factor A con dos niveles $\mathrm{y}$ el factor $\mathrm{B}$ con tres niveles, con 6 tratamientos y tres repeticiones. Los tratamientos del ensayo fueron: Temperatura de Almacenamiento al ambiente A1 $\left(16 \pm 4{ }^{\circ} \mathrm{C}\right.$ y HR de $\left.79 \%\right)$ y frío A2 $\left(6 \pm 2{ }^{\circ} \mathrm{C}\right.$ y HR de $\left.72-92 \%\right)$ y estado de madurez fruto tierno B1 (15-25\% color amarillo), fruto pintón B2 (50\% color amarillo) y fruto maduro B3 (75-90\% color amarillo). La madurez fisiológica de los frutos se determinó por su coloración amarillenta, realizando comparaciones mediante los soil color charts (Munsell 1973) (Figuras 1, 2 y 3).

Los frutos almacenados al ambiente, se llevaron a un cuarto con claridad suficiente y sobre una mesa se colocaron las canastillas plásticas que contenían los frutos, distribuidas de acuerdo al ensayo, se sometieron a una temperatura de $16 \pm 4{ }^{\circ} \mathrm{C}$ (empleando un termómetro escala $0-100{ }^{\circ} \mathrm{C}$. y a una humedad relativa promedio de $79 \%$ (utilizando un termohigrómetro de pared con un margen de medida de 0 a $60{ }^{\circ} \mathrm{C}$ y de 5 a $100 \%$ de HR.), durante los 26 días, como tiempo máximo de la evaluación. Para el almacenamiento al frío se utilizó una refrigeradora a temperatura de $6 \pm 2{ }^{\circ} \mathrm{C}$ y una humedad relativa de $72-92 \%$. Se controló estos dos parámetros 2 veces por semana, revisando a la vez la circulación de aire y el espacio entre las bandejas para que exista una ventilación adecuada.

Para la caracterización de los frutos de pitahaya, de cada tratamiento se tomaron 10 frutas al azar dos veces por semana durante 26 días (que duraron las evaluaciones del ensayo, aaproximadamente tiempo de la vida útil de la fruta), para realizar determinaciones de: pérdida de peso de la fruta (PP), contenido de sólidos solubles totales (SST), $\mathrm{pH}$, acidez titulable (AT) e índice de madurez (IM). Determinación de la pérdida de peso del fruto (PP). Utilizando una balanza digital con precisión de 0.1 y una capacidad máxima de 400 g. se determinó pesando tres frutos tomados al azar por tratamiento, efectuando lecturas periódicas del mismo dos veces por semana. La pérdida de peso del fruto se obtuvo 
por diferencia de las lecturas entre el valor inicial y el valor tomado en cada determinación y su resultado se expresó en porcentaje de pérdida de peso.

Determinación de sólidos solubles Totales (SST). Se llevó a cabo utilizando un refractómetro marca Abbe $\mathrm{N}$ 2933. La muestra de pulpa fue homogenizada (en una licuadora) y se midieron los grados Brix, con un Brixómetro marca Erma con escala de $0-50 \quad \%$ y estandarizando a $20{ }^{\circ} \mathrm{C}$ manteniendo durante toda la operación, de acuerdo (AOAC 1984 metodo 22024).

Determinación del pH. Mediante el uso de un pHmetro modelo $200 \mathrm{P}$ (estr. con buffer 10), previamente calibrada con una solución buffer de $\mathrm{pH}$ 10. Se determinó en la pulpa. (AOAC 1984 norma 22024).

Acidez Titulable (AT). Su determinación se realizó por titulación, siguiendo el procedimiento para jugos que detalla la norma (INEN 381 1986), el resultado se expresó en gramos de ácido cítrico/1000 $\mathrm{cm}^{3}$. Se tomaron $10 \mathrm{~cm}^{3}$ de pulpa de pitahaya previamente homogeneizada, utilizando una licuadora se colocaron en un frasco erlenmeyer de $250 \mathrm{~cm}^{3}$, se agregaron de 3 a 5 gotas de fenolftaleina como indicador, se llenó la bureta con hidróxido de sodio $0.1 \mathrm{~N}$ encerado, dejando caer lentamente la solución alcalina dentro del erlenmeyer y se agitó permanentemente hasta la aparición de una coloración rosada persistente.

Indice de Madurez (IM). Se calculó mediante la relación entre los sólidos solubles totales ( ${ }^{\circ}$ Brix) y la Acidez de la Fruta (g ácido cítrico/ $1000 \mathrm{~cm}^{3}$ ).

Para el análisis estadístico se empleó el programa el software estadístico versión 2014 InfoStat/L (Di Rienzo 2010), con la finalidad de establecer las diferencias entre tratamientos, llevándose a cabo un Analisis de Varianza (ANOVA) y la prueba de 111
Tukey al 5\% para la comparación entre los diferentes tratamientos.

\section{Resultados}

Pérdida de peso (PP). De forma general no se observaron diferencias significativas entre los diferentes tratamientos en cuanto a las variables de almacenamiento, al ambiente (promedio $17{ }^{\circ} \mathrm{C}$ ) y frío $\left(6 \pm 2{ }^{\circ} \mathrm{C}\right)$ y al estado de madurez (Figura 4$)$.

\section{Figura 4 Variación del peso en pitahaya en los tres estados de madurez (B1, B2 y B3) almacenadas a temperatura ambiente (A1) y frío (A2) hasta los 26 días}

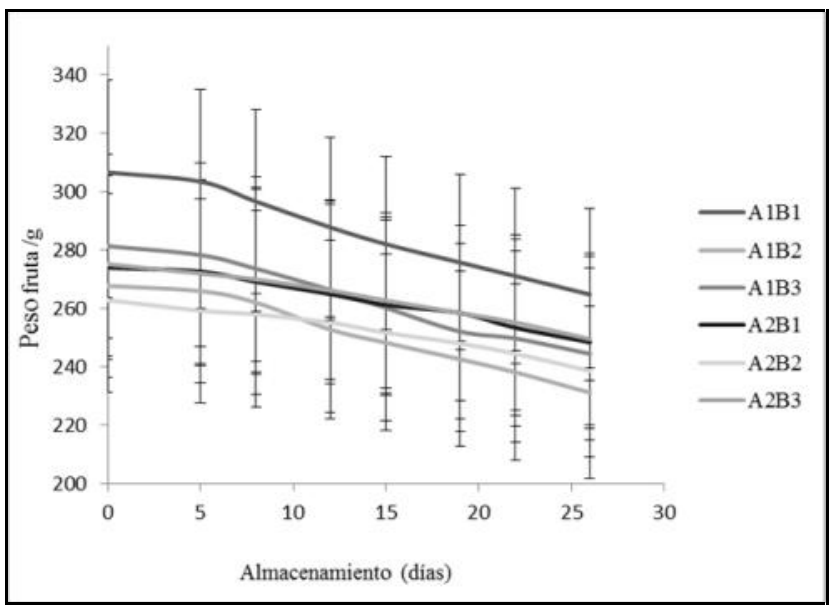

Sólidos Solubles Totales (SST). No se observaron diferencias significativas en cuanto a la concentración de sólidos solubles totales ( ${ }^{\circ}$ Brix) en los tratamientos almacenados al ambiente y al frío ni entre los diferentes estados de madurez (Figura 5). Tampoco se observó una tendencia concreta, ya que existían mediciones en las que aumentaban y otras disminuían.

pH (Potencial de Hidrógeno). El pH siguió un comportamiento diferente entre los distintos tratamientos de almacenamiento y grado de madurez (Figura 
6). El pH influye de forma importante en la calidad, ya que un aumento del mismo puede deteriorar la fruta por el desarrollo de microorganismos.

Figura 5 Variación de Sólidos Solubles Totales ( ${ }^{\circ} \mathrm{Brix}$ ) en pitahaya en los tres estados de madurez (B1, B2 y B3) almacenadas a temperatura ambiente (A1) y frío (A2) hasta los 26 días

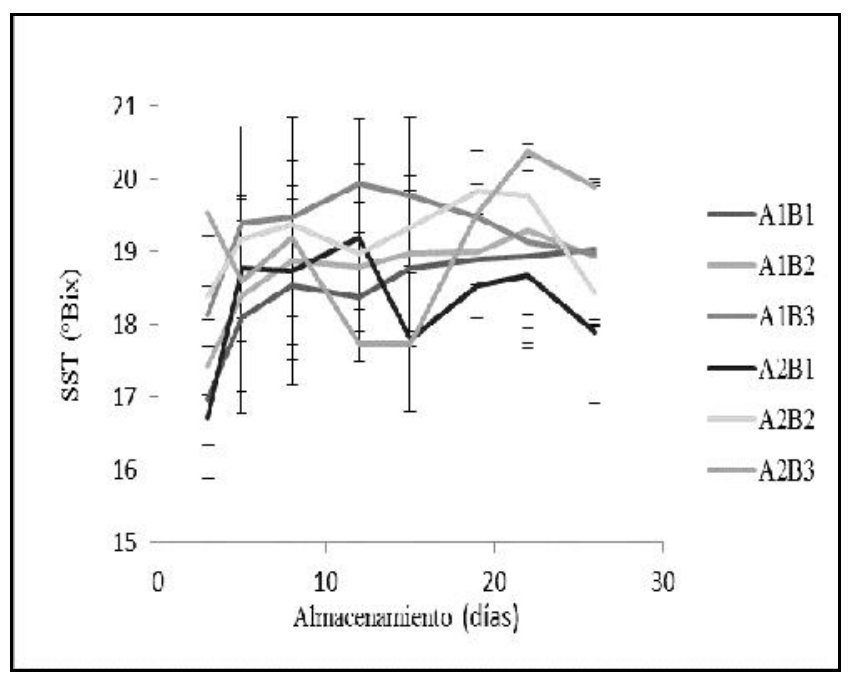

Figura 6 Variación de los valores de $\mathrm{pH}$ de fruta de Pitahaya en los tres estados de madurez (B1, B2 y B3) almacenadas a temperatura ambiente (A1) y frío (A2) hasta los 26 días

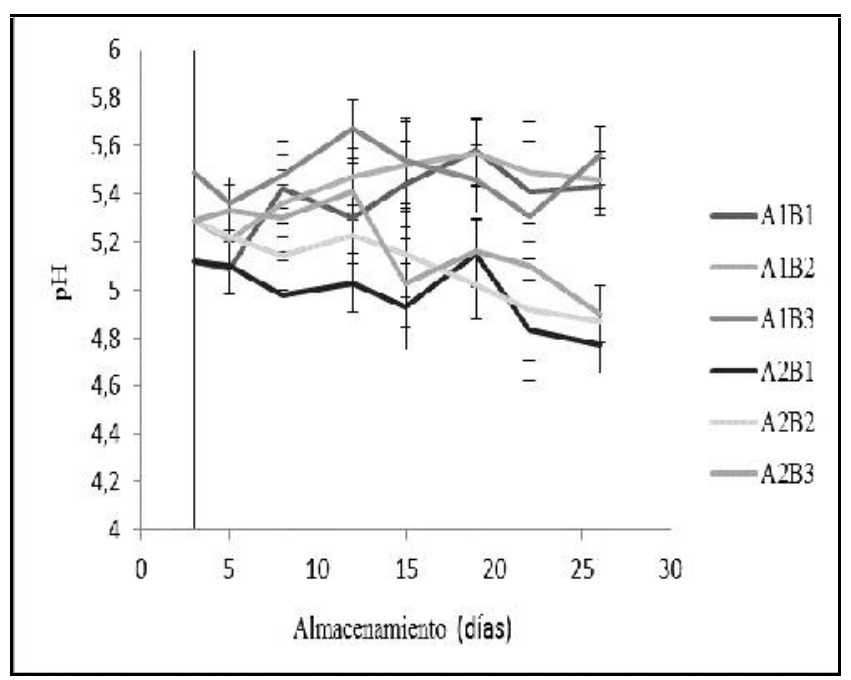

Figura 7 Variación de la acidez titulable de fruta pitahaya en los tres estados de madurez (B1, B2 y B3) almacenadas a temperatura ambiente (A1) y frío (A2) hasta los 26 días

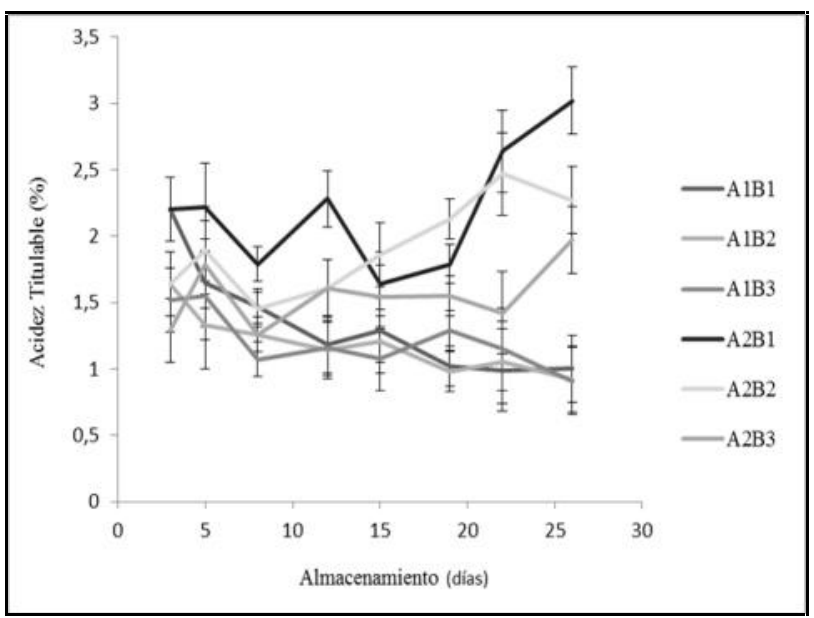

Figura 8 Variación de índice de madurez de fruta pitahaya en los tres estados de madurez (B1, B2 y B3) almacenadas a temperatura ambiente (A1) y frío (A2) hasta los 26 días

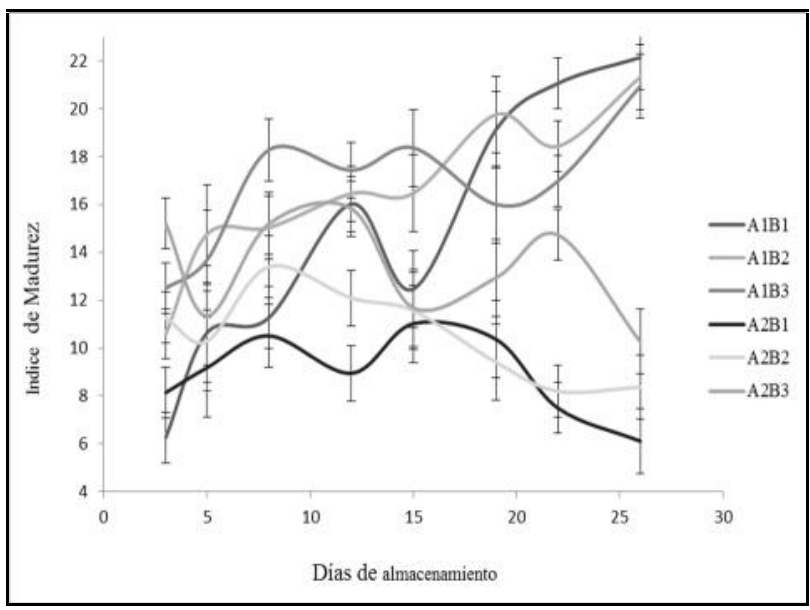

Acidez Titulable (AT). La acidez es el resultado de los ácidos orgánicos, que influyen en diferentes parámetros de la calidad de la fruta, como color, sabor y olor. En cuanto a la acidez titulable, a medida que avanzó el estado de madurez, en los frutos almacenados al ambiente, disminuye la acidez. Caso contrario ocurrió en los frutos almacenados al frío, 
donde se observa un incremento de la misma (Figura 7).

Índice de Madurez (IM). Las plantas almacenadas al ambiente tuvieron un aumento en el índice de madurez observado a partir del día 19 de almacenamiento (Figura 8), causado fundamentalmente por un descenso en la acidez titulable.

\section{Discusión}

Pérdida de peso (PP). A los 26 días, las frutas de pitahaya a temperatura ambiente perdieron un promedio de $12.5 \%$ de peso en los tres estados de madurez, mientras que los frutos almacenados en frío, mostraron una pérdida promedio del $8.2 \%$. Según (Osuna-Enciso et al. 2011) la pérdida de peso acumulada presentó cambios significativos ( $\mathrm{P} \unlhd$.05) durante el tiempo de almacenamiento y entre estados de madurez. Los frutos cosechados en madurez inicial tuvieron la mayor pérdida de peso después de 12 días (7.8\%), mientras que los cosechados con madurez media y completa registraron 6.1 y $5.6 \%$, respectivamente, sin diferencia entre éstos. (Magaña-Benítez et al. 2013) también encontraron pérdidas de peso menor del $8 \%$ evaluado el día cero de la cosecha hasta los seis días.

Sólidos Solubles Totales (SST). De forma general los valores de SST variaron entre 16.9 y $20.3^{\circ}$ Brix. Los resultados obtenidos concuerdan con los de Magaña-Benítez et al. 2013) que encontraron que los ${ }^{\circ}$ Brix de los SST no varían significativamente desde el día cero hasta el día seis de su evaluación. pH (Potencial de Hidrógeno). A partir del día 12 se produjo un aumento en el $\mathrm{pH}$ de las muestras almacenadas a temperatura ambiente con respecto a las almacenadas en frío. Un aumento en el pH de muestras de pitahaya en almacenamiento también ha sido observado por (Rodríguez-Rodríguez et al. 2005) 113 donde los frutos almacenados a $8{ }^{\circ} \mathrm{C}$ y $19{ }^{\circ} \mathrm{C}$ aumento el $\mathrm{pH}$.

Además, el día 12 el tratamiento con mayor grado de madurez (A1B3) fue significativamente superior al de menor grado de madurez (A2B1), como ocurrió en los estudios de (Rodríguez-Rodríguez et al. 2005). Los valores de $\mathrm{pH}$ de todas las muestras del ensayo variaron entre 4.90 y 5.67. (Magaña et al.2013) en su evaluación del pH determinó un incremento de 4 a 7 desde el dia cero (cosecha) hasta el sexto día evaluado.

Acidez Titulable (AT). Con respecto al grado de madurez en los tratamientos almacenados al ambiente, se observa que el tratamiento tierno (A1B1) perdió un $35.5 \%$ de la acidez total, mientras que el tratamiento más maduro (A1B3) perdió una mayor proporción, llegando al 59.9\%. RodríguezRodríguez et al. (2005) encontró que la acidez titulable decreció, pasando de $2.54 \%$ a $1.50 \%$ y 3.02 $\%$ a $1.29 \%$ en estado 3 de maduración a $19{ }^{\circ} \mathrm{C}$ y 8 ${ }^{\circ} \mathrm{C}$, respectivamente.

(Magaña-Benítez et al. 2013) obtuvieron una disminución de los valores de acidez entre 0.5 y 0.1 (Osuna-Enciso et al. 2011) observaron que los frutos cosechados en madurez inicial tuvieron una menor pérdida de acidez que los frutos con madurez mediana y completa.

Índice de Madurez (IM). En cuanto al estado de madurez en los diferentes tratamientos, el día 8 se observó un menor IM en los tratamientos de menor grado de madurez (tiernos), con respecto a los de mayor grado de madurez (madurez total), tanto a temperatura ambiente como en frío. Todos los tratamientos variaron entre un IM de 6.2 y 22.1. Los resultados de este estudio son similares a los obtenidos por Rodríguez-Rodríguez et al. (2005) los frutos a $8{ }^{\circ} \mathrm{C}$ y $19{ }^{\circ} \mathrm{C}$, presentaron aumento, pasando de estado 3 a 5 y de 5 a 6 al final del almacenamiento. 
Del presente trabajo de investigación se concluye que las mejores condiciones de almacenamiento de pitahaya amarilla son a temperatura en frío y madurez tierna (A2B1), observándose un menor aumento del $\mathrm{pH}$ y una mayor acidez titulable. Otros factores como la pérdida de peso o el contenido en sólidos solubles totales no sufren cambios entre los tratamientos. Las frutas almacenadas al frío tuvieron un menor índice de madurez, su maduración fisiológica disminuyó en el tiempo, y esto provoca que la vida útil del fruto se extienda, consideraciones que deben tomar en cuenta los agricultores para el manejo postcosecha de la fruta.

\section{Conflictos de intereses}

Esta investigación se realizó en los laboratorios de la Facultad de Alimentos de la Universidad Técnica de Ambato Ecuador y no existe ningún tipo de conflicto de intereses.

\section{Agradecimientos}

Los autores agradecen a la Universidad Técnica de Ambato Facultad de Ciencias Agropecuarias, por el apoyo técnico, científico y logístico realizado a la presente investigación.

\section{Literatura citada}

Aguilar GE. Evaluación de tres enraizantes y dos tamaños de cladodios en la propagación asexual de pitahaya amarilla cereus triangularis (L.) Haw., en Yantzaza). [Tesis de Licenciatura]. Universidad Nacional de Loja; 2015.

AOAC. Official Methods of Analysis. Association of Official Analytical Chemist. Washington D.C. USA. $14^{\mathrm{a}}$ ed. 1984. Disponible en: http://catarina.udlap.mx/u dl_a/tales/document os/lia/robinson_1_a/bibliografia.pdf.

Balderas V, Palafox L, Castro AS, Saucedo C. Evaluación de las propiedades físicas y calidad organoléptica y nutricional de frutos de pitaya (Stenocereus pruinosus). Rev Iberoam Cienc 2016;3(7):86-92.

Balois-Morales R, Peña-Valdivia CB, Arroyo-Peña VB. Síntomas y sensibilidad al daño por frío de frutos de pitahaya (Hylocereus undatus Haw.) durante la postcosecha. Agrociencia 2013;47(8):795-813.

Corrales J. Caracterización postcosecha, aprovechamiento e industrialización de pitayas y pitahayas. [Reporte de investigación 65 en el internet]. 2002 [citado 2017 Oct 25]; Disponible en: file:///C:/Users/Usuario/Downloads/ Caracterizacinpostcosechaaprovechamientoeindustrializacindepitayasypitahayas.pdf.

Di Rienzo J, Balzarini M, Gonzalez L, Casanoves F, Tablada M, Robledo CW. InfoStat/L software estadístico versión 2014. Universidad Nacional de Córdoba (FCA-UNC) [Internet]. 2010 [citado 2017 Oct 23]; Disponible en: http://www .infostat.com.ar/.

Dueñas YM, Narváez CE, Restrepo LP. El choque térmico mejora la aptitud al almacenamiento refrigerado de pitaya amarilla. Agron Colomb 2009;27(1):105-10.

Huachi L, Yugsi E, Paredes MF, Coronel D, Verdugo K, Pablo Coba P. Desarrollo de la pitahaya (Cereus sp.) en Ecuador. Granja 2015; 22(2):50-8.

Magaña-Benítez W, Baldín AM, Corrales GJ, Saucedo VC, Sauri E. Variaciones bioquímicasfisiológicas y físicas de las frutas de pitahaya (Hylocereus undanatus) almacenadas en ambiente natural. Rev Iber Tecnología Postcosecha 2013;4(1):21-30. 
Munsell. Soil color charts. [Internet]. 1973 [Citado 2017 Oct 25]; Macbeth, a Division of Baltimore, MD : Munsell Products, MacBeth Color \& Photometry Division of Kollmorgen Corp. Disponible en: http://www.worldcat.org/ title/munsell-soil-color-charts/oclc/70385250 \&referer=brief_results.

NTE (Norma Técnica Ecuatoriana) INEN 0381: Conservas vegetales. Determinación de acidez titulable. Método potenciométrico de referencia [Internet]; 1986 [Citado 2017 Oct 25]; Disponible en: https://archive.org/stream/ec.nte $.0381 .1986 /$ ec .nte.0381.1986 djvu.txt.

Osuna-Enciso T, Ibarra-Zazueta ME, Muy-Rangel MD, Valdez-Torres JB, Villareal-Romero M, Hernández-Verdugo S. Calidad postcosecha de frutos de pitahaya (Hylocereus undatus Haw.) cosechados en tres estados de madurez. Rev Fitotec Mex 2011;34(1):63-72.
Pro Ecuador (Instituto de promoción de exportaciones e inversiones) Análisis sectorial pitahaya [Análisis sectorial en el internet]. 2016. [citado 2017 Oct 25]; p.12. Disponible en: http:// www.proecuador.gob.ec/wp-content/uploads /2016/05/PROEC_AS2016_PITAHAYA.pdf. Rodríguez-Rodríguez DA, Patiño-Gutierrez MP, Miranda-Lasprilla D, Fischer G, Galvis-Vane gas JA. Efecto de dos índices de madurez y dos temperaturas de almacenamiento sobre el com portamiento en poscosecha de la pitahaya amarilla (Selenicereus megalanthus Haw.). Rev Fac Nal Agr Medellín 2005;58(2): 2837-57. 